\title{
INFLUÊNCIA DA ADIÇÃO DE ÁCIDO ASCÓRBICO NA COMPOSIÇÃO E NAS CARACTERÍSTICAS SENSORIAIS DA GARAPA PARCIALMENTE CLARIFICADA-ESTABILIZADA E ESTOCADA SOB REFRIGERAÇÃO
}

\author{
PATRICIA PRATI * \\ ROBERTO HERMÍNIO MORETTI ** \\ HELENA MARIA CARDELLO ***
}

\begin{abstract}
Testou-se o efeito de três distintas concentrações de ácido ascórbico (50 ppm - amostra 1; 125 ppm - amostra 2 e 200 ppm - amostra 3) sobre a qualidade sensorial da garapa parcialmente clarificada-estabilizada, submetida a posterior acidificação e adicionada de conservante antes da pasteurização $\left(82^{\circ} \mathrm{C} / 15 \mathrm{seg}\right)$. Após o tratamento térmico, a bebida foi resfriada, embalada em garrafas PET e armazenada sob refrigeração $\left(4-6^{\circ} \mathrm{C}\right)$ por dois meses. Além da avaliação sensorial, em cada tempo de armazenamento foram realizadas análises físicoquímicas $\left(\mathrm{pH},{ }^{\circ}\right.$ Brix, acidez, ratio e teor de ácido ascórbico) do produto. Verificou-se diminuição nos teores de vitamina $\mathrm{C}$ nas três amostras, durante o prazo de armazenamento. Apesar disso, os resultados do Teste de Aceitação indicaram que as três amostras mantiveram sua qualidade sensorial durante o período de estocagem. Elegeu-se a amostra 2 como a melhor devido ao nível intermediário de ácido ascórbico, tornando o produto menos dispendioso.
\end{abstract}

PALAVRAS-CHAVE: CANA-DE-AÇÚCAR-ANÁLISE SENSORIAL; GARAPA.

* Doutoranda em Tecnologia de Alimentos, Faculdade de Engenharia de Alimentos (FEA), Universidade Estadual de Campinas (UNICAMP), Campinas-SP, Brasil (e-mail: pprati00@yahoo.com).

** Professor Titular, Departamento de Tecnologia de Alimentos, FEA, UNICAMP, Campinas-SP (e-mail: moretti@fea.unicamp.br).

*** Professora Doutora, Departamento de Alimentos e Nutrição, FEA, UNICAMP, Campinas-SP, Brasil (e-mail: hellini@fea.unicamp.br). 


\section{INTRODUÇÃO}

O caldo-de-cana (ou garapa) é considerado produto altamente nutritivo, de sabor agradável e barato. É comercializado normalmente na rua, por vendedores ambulantes que possuem moendas para extração. A maioria desses vendedores não dispõe de instalações compatíveis, assim como de instrução adequada para obtenção de produto em condições higiênico-sanitárias apropriadas (SOCCOL et al., 1990).

YUSOF et al. (2000) citam que a garapa é muito popular na Malásia, sendo considerada como bebida agradável ao paladar e que sacia a sede. Sua comercialização no país estende-se desde restaurantes simples de beira de estrada até restaurantes de hotéis de alta classe. Tal fato revela a lucratividade da produção de caldo-de-cana e a importância de desenvolver processos que possam conservar a qualidade do produto fresco durante sua estocagem.

A garapa pode ser considerada como suco da cana. A qualidade dos sucos é influenciada por fatores que comprometem suas características químicas (composição), físicas (turbidez, separação de fases sólido/ líquido), organolépticas (aroma, sabor, cor, consistência) e nutricionais (vitaminas). Tais fatores, juntamente com as alterações que ocorrem durante o acondicionamento, distribuição e estocagem irão influenciar a vida-de-prateleira do produto (GRAUMLICH et al., 1986).

Os processos de conservação são baseados na eliminação total ou parcial dos agentes que alteram os produtos, na modificação ou na supressão de um ou mais fatores essenciais à manifestação desses agentes. Como regra geral, os melhores métodos devem garantir satisfatória conservação com menor alteração das características naturais dos produtos. Após os tratamentos, a conservação é assegurada pelo uso de embalagem apropriada (EVANGELISTA, 1994; FENNEMA, 1975).

Os sucos prontos para beber, pasteurizados e comercializados sob refrigeração, constituem segmento que desperta o interesse dos fabricantes de sucos devido à preferência do consumidor moderno pelo alimento refrigerado.

O mercado brasileiro é altamente exigente quanto ao sabor/odor natural 
dos sucos, geralmente detectando alterações devido a tratamentos térmicos intensos (ALVES e GARCIA, 1993; CORRÊA NETO, 1998). Os alimentos refrigerados são submetidos a processamentos térmicos mais brandos e, portanto, suas características organolépticas permanecem próximas às do produto fresco.

Os fatores químicos que afetam a qualidade dos sucos normalmente são de natureza oxidativa. A oxidação ocorre com a vitamina $C$ (ácido ascórbico) e com os compostos responsáveis pelo aroma e sabor do suco, alterando significativamente suas características sensoriais e nutricionais. As reações oxidativas dependem das condições de processo (tratamento térmico), da presença de oxigênio, da embalagem, da relação tempo/temperatura de estocagem, da influência da luz e da presença de catalizadores (Fe, Cu) (ALVES e GARCIA, 1993; GRAUMLICH, et al., 1986; TOCCHINI, 1985).

O oxigênio exerce grande influência na qualidade e estabilidade de sucos de frutas, podendo estar presente no produto de forma dissolvida no espaço livre da embalagem (oxigênio residual) ou ainda ser permeado por meio dessa. Sendo assim, a desaeração contribui para a retenção do ácido ascórbico, do aroma e do sabor durante o processamento dos sucos (GRAUMLICH et al., 1986).

A oxidação do ácido ascórbico implica em perdas nutricionais e também no escurecimento do suco (browning), pois resulta na produção de compostos com radical carbonila que (reagindo com grupos amino e por polimerização) produzem pigmentos escuros (SHAW e MOSHONAS, 1991). Outras formas de escurecimento (browning) nãoenzimático podem ocorrer pela reação de Maillard ou pela oxidação de açúcares, com formação de aldeídos ativos (teoria do aldeído ativo) (SIMÃO, 1985). Existe ainda a perda da cor característica do produto pela oxidação de pigmentos (carotenóides, principalmente), fato comum em alimentos contendo carboidratos.

A luz exerce importante papel na destruição da vitamina $C$ em suco de laranja pasteurizado e envasado a quente, evidenciando efeito catalítico da luz sobre a oxidação aeróbica do ácido ascórbico (MARTIN et al., 1995). Todos esses fatores evidenciam a importância de se adicionar antioxidante à garapa clarificada e acidificada, já que o produto será processado e estocado sob refrigeração por dois meses. 
O ácido ascórbico, além de sua propriedade antioxidativa, contribui para melhorar o valor nutritivo das bebidas (vitamina). Trata-se de elemento de grande importância como substância tampão em processos de oxirredução, que interfere no metabolismo do ferro e dos glicídios. A vitamina $\mathrm{C}$, graças ao seu comportamento químico, é utilizada na preservação de alimentos susceptíveis à oxidação, tais como os sucos de frutas. A forma de atuação do ácido ascórbico como antioxidante ocorre pela sua combinação com o oxigênio (ABREU e SCHMITZ, 1971).

Os conservantes são substâncias que impedem ou retardam alterações provocadas por microrganismos e/ou enzimas em alimentos (SIMÃO, 1985). Os parabenos (compostos químicos sintéticos) compreendem grupo de agentes antimicrobianos, largamente empregados em cosméticos e produtos farmacêuticos. Posteriormente, passaram a ser usados em alimentos devido ao $\mathrm{pH}$, que permite faixa de atividade muito mais ampla que o benzoato (LEITÃO, 1973).

O objetivo da pesquisa foi estudar três concentrações de ácido ascórbico a serem adicionadas como antioxidante à garapa clarificadaestabilizada (contendo também conservador), pasteurizada e estocada em garrafas de polietileno tereftalato (PET) sob refrigeração.

\section{MATERIAL E MÉTODOS}

\subsection{MATERIAL}

\subsubsection{Matéria-prima}

Empregou-se caldo-de-cana-de-açúcar (Saccharum spp) da variedade RB72-454, usada para comercialização de garapa na região de Piracicaba. A extração foi efetuada em moenda elétrica (Modelo STN30 / 270 rpm) na Planta Piloto do Setor de Frutas, Hortaliças e Produtos Açucarados do Departamento de Tecnologia de Alimentos da Faculdade de Engenharia de Alimentos, Universidade de Campinas.

\subsubsection{Equipamentos, vidrarias, reagentes e embalagens}

Foram empregados equipamentos e vidrarias tanto de uso comum em laboratório como específicos para as análises físicas, químicas e 
sensoriais realizadas. Reagentes específicos foram usados para as análises físicas e químicas. O policloreto de alumínio (PAC) do tipo "Panclar P-1010" foi o único agente de clarificação empregado no processo.

Como antioxidante utilizou-se o ácido ascórbico, produto naturalmente encontrado na natureza e como conservador o parabeno (pelas vantagens que apresenta e em concentração previamente estabelecida).

Foram utilizadas embalagens do tipo PET com capacidade para 500 $\mathrm{mL}$, incolores e transparentes, fornecidas gentilmente pela empresa Braspet (localizada no município de Louveira - região de Campinas $\mathrm{SP})$.

\subsection{MÉTODOS}

O estudo da vida-de-prateleira do produto baseou-se em determinações físico-químicas e sensoriais, estabelecendo-se a concentração mais apropriada do aditivo. Testes microbiológicos foram realizados, preliminarmente, para verificar em quanto tempo ocorre a deterioração do produto.

\subsubsection{Matéria-prima}

Previamente à extração, os colmos tiveram sua casca removida manualmente com faca, sendo sanitizados com solução contendo 10 ppm de Cloro Ativo. Após 10 minutos de contato com a solução, o material foi enxaguado com água corrente tratada. Procedeu-se da mesma forma para a sanitização da moenda empregada nessa operação.

O caldo extraído foi submetido à clarificação-estabilização conforme estabelecido por PRATI e MORETTI (2002). O caldo foi aquecido em banho-maria $\left(65^{\circ} \mathrm{C} / 50 \mathrm{~min}\right)$, alcalinizado com $\mathrm{Ca}(\mathrm{OH})_{2}$ até $\mathrm{pH} 8$, adicionado de 60 ppm de policloreto de alumínio e decantado por 40 minutos. O sobrenadante foi separado com o uso de bomba de vácuo (Modelo TE-058). 


\subsubsection{Produto processado}

A garapa clarificada foi processada conforme descrito na Figura 1, sendo testadas três concentrações de ácido ascórbico (200, 125 e 50 ppm) que correspondem a 3 tratamentos.

\section{FIGURA 1 - FLUXOGRAMA DA OBTENÇÃO DE CALDO-DE-CANA CLARIFICADO-ESTABILIZADO, ADICIONADO DE CONSERVANTE E ANTIOXIDANTE}

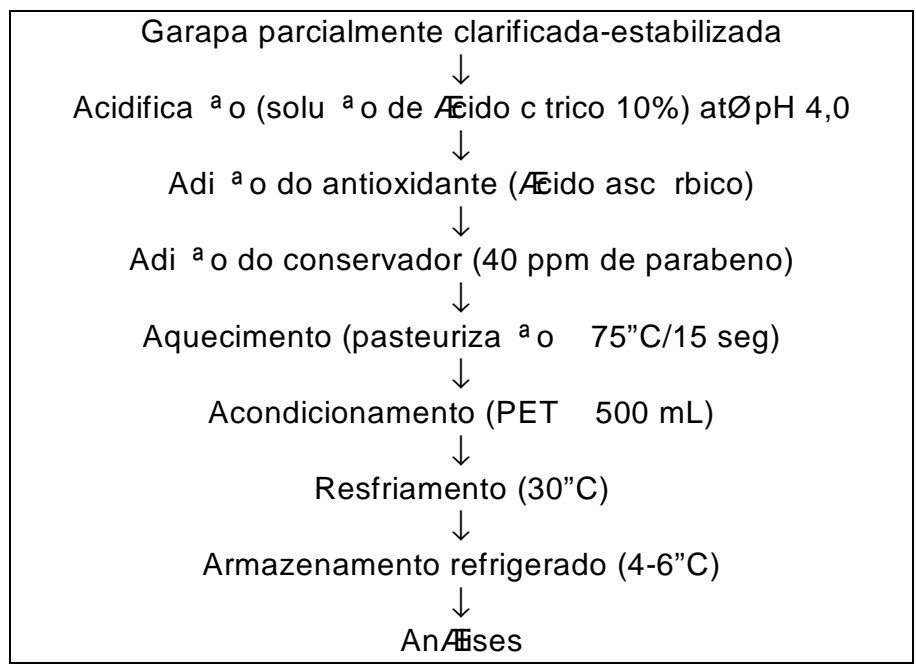

\subsubsection{Análise Sensorial}

Teste de Aceitação, mediante escala hedônica não-estruturada de 9 pontos (método afetivo), foi aplicado nos tempos 0, 15, 30, 45, 60 e 66 dias de armazenamento do produto, que correspondem a $0,25,50$, 75,100 e $110 \%$ do período total de armazenamento conforme recomenda a ASTM (1993).

Foram avaliados os atributos sensoriais de aparência, cor, aroma, sabor e impressão global do produto (MEILGAARD et al., 1987; STONE e SIDEL, 1993). 
A equipe sensorial foi composta por 31 julgadores não-treinados. As amostras (3) codificadas foram servidas em copos plásticos descartáveis, em volume padronizado de $50 \mathrm{~mL}$, em ambiente claro (mesa branca) e na forma de blocos completos casualizados. As amostras estavam acompanhadas de biscoito, copo d'água e da ficha de avaliação.

A análise sensorial permitiu estudar o efeito do tempo de armazenamento sobre a qualidade das amostras mediante Análise de Regressão dos resultados que foram submetidos à Análise de Variância (ANOVA) e ao Teste de Média de Tukey $(p \leq 0,05)(S A S, 1993)$.

\subsubsection{Análises físico-químicas}

Concomitantemente às avaliações sensoriais foram determinados o $\mathrm{pH}$, segundo metodologia do Instituto Adolfo Lutz (1976), o teor de sólidos solúveis (Brix), conforme método n.37.1.15 da AOAC (1997), a acidez total titulável (\% ácido cítrico), pelo método n.37.1.37 da AOAC (1997) e o teor de ácido ascórbico, segundo método n.43046 da AOAC (1984), modificado por BENASSI (1990). O ratio foi definido pela relação ${ }^{\circ}$ Brix/ acidez total titulável (ATT).

Os resultados das análises físico-químicas também foram submetidos à ANOVA e ao teste de média de Tukey $(p \leq 0,05)$, utilizando-se 0 pacote STATISTICA FOR WINDOWS 5.0 (1995). Posteriormente, elaborou-se gráfico com os dados dos teores de ácido ascórbico, ilustrando o comportamento desse componente no produto, com o decorrer do período de armazenamento.

Para definir a concentração mais adequada de antioxidante foram utilizados os resultados do teste de aceitação.

\section{RESULTADOS E DISCUSSÃO}

\subsection{ANÁLISE SENSORIAL}

Os atributos cor, sabor e impressão global das amostras foram considerados os mais relevantes para a pesquisa. Apenas esses resultados foram submetidos à Análise de Regressão durante o tempo de armazenamento e ao Teste de Tukey (a 5\% de probabilidade). 
Observando as Figuras 2 a 10, que ilustram a Análise de Regressão dos atributos cor, sabor e impressão global das três amostras analisadas, verifica-se que não houve correlação linear significativa $(p \leq 0,05)$ entre o tempo de armazenamento e os atributos sensoriais em questão. Portanto, não houve alteração na qualidade sensorial das três amostras durante o período de estocagem (66 dias).

\section{FIGURA 2 - ANÁLISEDEREGRESSÃOCONTENDOAEQUAÇÃODARETA E OVALOR DE R² PARA A AMOSTRA 1 (50 ppm)-ATRIBUTO COR}

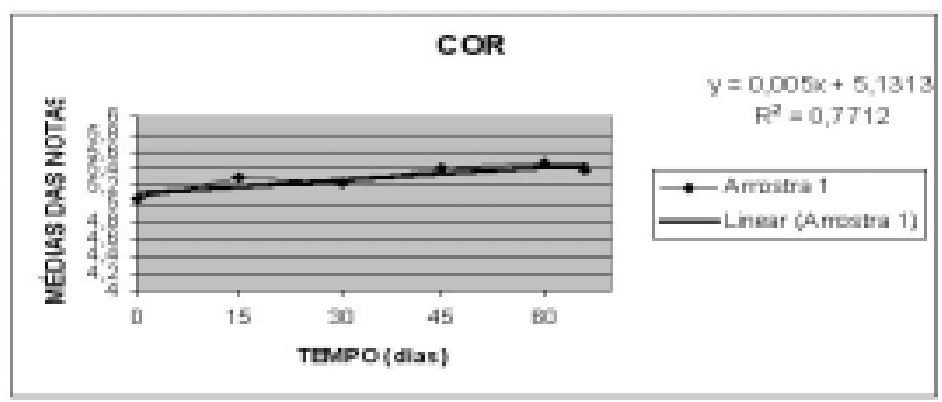

FIGURA 3 - ANÁLISEDEREGRESSÃOCONTENDOAEQUAÇÃODARETA E O VALOR DE R ${ }^{2}$ PARA A AMOSTRA 2 (125 ppm) ATRIBUTOCOR

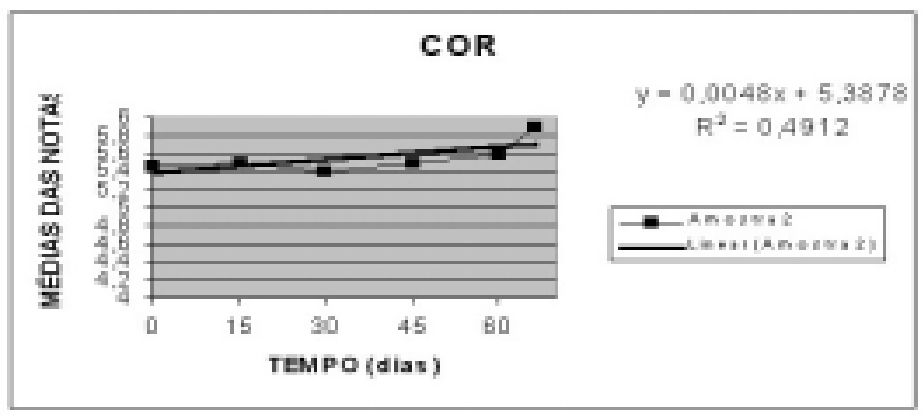


FIGURA 4 - ANÁLISEDEREGRESSÃOCONTENDOAEQUAÇÃODARETA E O VALOR DE R ${ }^{2}$ PARA A AMOSTRA 3 (200 ppm) ATRIBUTOCOR

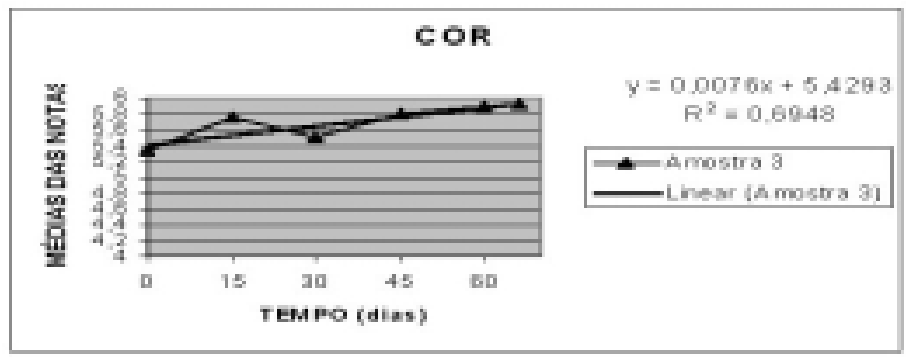

FIGURA 5 - ANÁLISEDEREGRESSÃOCONTENDOAEQUAÇÃODARETA EOVALOR DE R² PARA A AMOSTRA 1 (50 ppm)-ATRIBUTO SABOR

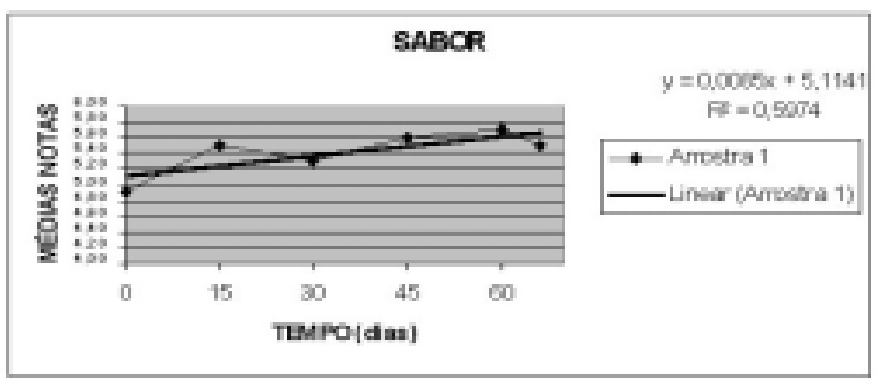

FIGURA 6 - ANÁLISEDEREGRESSÃOCONTENDOAEQUAÇÃODARETA E O VALOR DE R² PARA A AMOSTRA 2 (125 ppm) ATRIBUTOSABOR

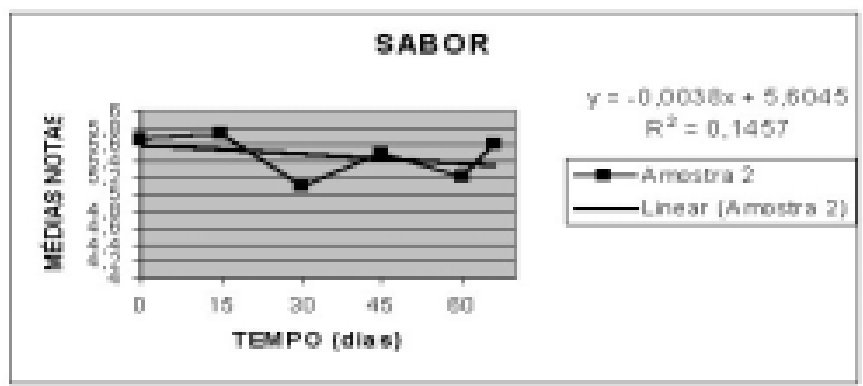


FIGURA 7 - ANÁLISEDEREGRESSÃOCONTENDOAEQUAÇÃODARETA E O VALOR DE R ${ }^{2}$ PARA A AMOSTRA 3 (200 ppm) ATRIBUTOSABOR

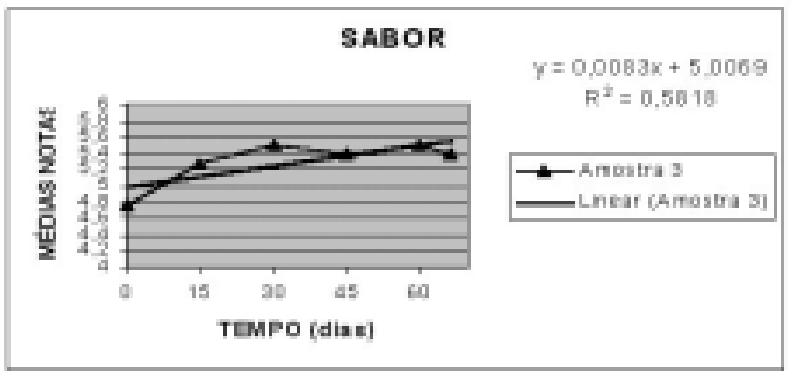

FIGURA 8 - ANÁLISEDEREGRESSÃOCONTENDOAEQUAÇÃODARETA E OVALOR DE R ${ }^{2}$ PARA A AMOSTRA 1 (50 ppm)-ATRIBUTO IMPRESSÃOGLOBAL

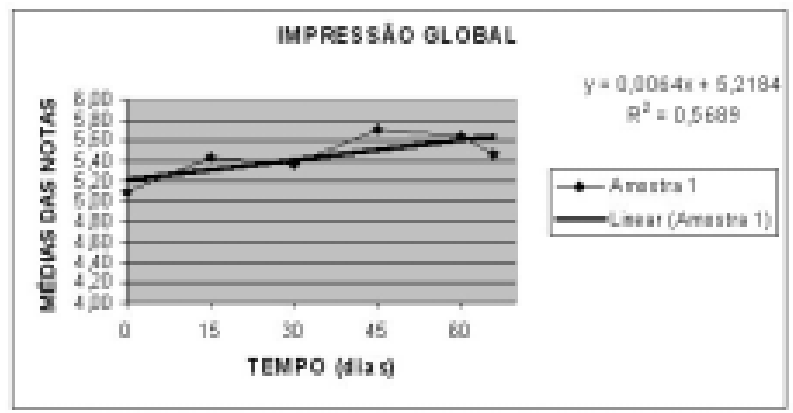

FIGURA 9 - ANÁLISEDEREGRESSÃOCONTENDOAEQUAÇÃODARETA E O VALOR DE R² PARA A AMOSTRA 2 (125 ppm) ATRIBUTOIMPRESSÃOGLOBAL

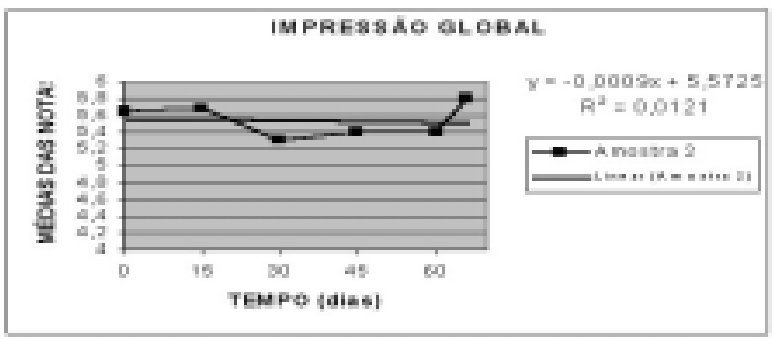


FIGURA 10 - ANÁLISEDEREGRESSÃOCONTENDOAEQUAÇÃODARETA E O VALOR DE R ${ }^{2}$ PARA A AMOSTRA 3 (200 ppm) ATRIBUTOIMPRESSÃOGLOBAL

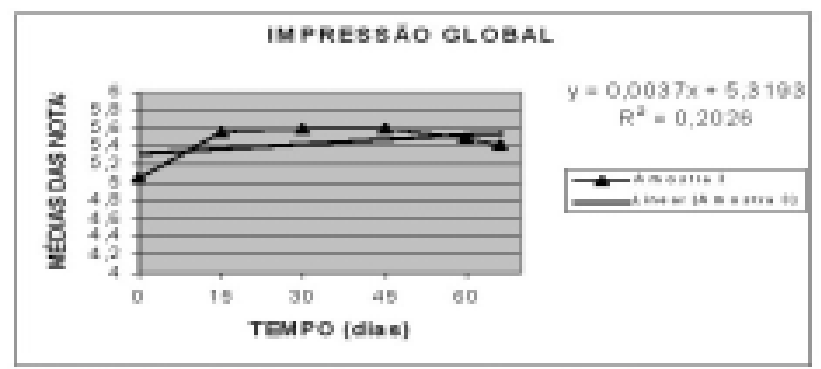

A Tabela 1 mostra as médias das notas atribuídas às amostras durante a estocagem.

\section{TABELA 1 - MÉDIAS DAS NOTAS DAS AMOSTRAS QUANTO AOS ATRIBUTOS COR, SABOR E IMPRESSÃO GLOBAL NOS DIFERENTES TEMPOS DE ARMAZENAMENTO}

\begin{tabular}{|c|c|c|c|c|c|c|}
\hline \multirow[t]{2}{*}{ TEMPO } & \multicolumn{2}{|c|}{ COR } & \multicolumn{2}{|c|}{ SABOR } & \multicolumn{2}{|c|}{ IMPRESS^ O GLOBAL } \\
\hline & Amostra & MØdia & Amostra & Media & Amostra & MØdia \\
\hline \multirow[t]{3}{*}{0} & 2 & $5.47^{a}$ & 2 & $5.67^{\mathrm{a}}$ & 2 & $5.66^{\mathrm{a}}$ \\
\hline & 3 & $5.34^{a}$ & 1 & $4.91^{b}$ & 1 & $5.09^{\mathrm{a}}$ \\
\hline & 1 & $5.06^{a}$ & 3 & $4.76^{b}$ & 3 & $5.05^{\mathrm{a}}$ \\
\hline \multirow[t]{3}{*}{15} & 3 & $5.76^{a}$ & 2 & $5.73^{\mathrm{a}}$ & 2 & $5.69^{\mathrm{a}}$ \\
\hline & 2 & $5.53^{a}$ & 1 & $5.49^{\mathrm{a}}$ & 3 & $5.56^{\mathrm{a}}$ \\
\hline & 1 & $5.30^{a}$ & 3 & $5.27^{\mathrm{a}}$ & 1 & $5.43^{\mathrm{a}}$ \\
\hline \multirow[t]{3}{*}{30} & 3 & $5.49^{a}$ & 3 & $5.54^{a}$ & 3 & $5.58^{\mathrm{a}}$ \\
\hline & 2 & $5.38^{a}$ & 1 & $5.26^{\mathrm{a}}$ & 1 & $5.36^{\mathrm{a}}$ \\
\hline & 1 & $5.24^{a}$ & 2 & $5.11^{\mathrm{a}}$ & 2 & $5.30^{\mathrm{a}}$ \\
\hline \multirow[t]{3}{*}{45} & 3 & $5.81^{a}$ & 1 & $5.57^{\mathrm{a}}$ & 1 & $5.71^{a}$ \\
\hline & 2 & $5.46^{a}$ & 2 & $5.46^{a}$ & 3 & $5.57^{\mathrm{a}}$ \\
\hline & 1 & $5.41^{a}$ & 3 & $5.35^{\mathrm{a}}$ & 2 & $5.44^{\mathrm{a}}$ \\
\hline \multirow[t]{3}{*}{60} & 3 & $5.90^{a}$ & 1 & $5.72^{\mathrm{a}}$ & 1 & $5.64^{\mathrm{a}}$ \\
\hline & 2 & $5.59^{a b}$ & 3 & $5.46^{a}$ & 3 & $5.45^{\mathrm{a}}$ \\
\hline & 1 & $5.46^{b}$ & 2 & $5.22^{\mathrm{a}}$ & 2 & $5.41^{\mathrm{a}}$ \\
\hline \multirow[t]{3}{*}{66} & 3 & $5.92^{2}$ & 2 & $5.62^{\mathrm{a}}$ & 2 & $5.80^{\mathrm{a}}$ \\
\hline & 2 & $5.91^{a}$ & 1 & $5.50^{\mathrm{a}}$ & 1 & $5.47^{\mathrm{a}}$ \\
\hline & 1 & $5.39^{b}$ & 3 & $5.42^{a}$ & 3 & $5.39^{\mathrm{a}}$ \\
\hline
\end{tabular}

Médias seguidas pela mesma letra minúscula não diferem entre si ao nível de $5 \%$ de significância. 
Para o atributo cor, os resultados obtidos nos tempos 60 e 66 dias diferiram dos demais, por tratar-se de equipe de julgadores nãotreinados. Tal diferença não é relevante pela ausência de correlação linear significativa $(p \leq 0,05)$ entre os tempos e a cor das amostras. Sendo assim, é possível afirmar que as amostras não diferiram entre si quanto ao atributo cor durante o tempo de estocagem.

Em relação ao sabor, a diferença observada no tempo 0 também é justificada pela equipe de julgadores não ser treinada. A Análise de Regressão não revelou correlação linear significativa $(p \leq 0,05)$ entre os tempos e o sabor das amostras. Pode-se, então, afirmar que as amostras não diferiram entre si quanto ao atributo sabor nos diferentes tempos de estocagem.

Para o atributo impressão global, também é possível afirmar que as amostras não diferiram entre si durante o tempo de armazenagem.

\subsection{DETERMINAÇÕES FÍSICO-QUÍMICAS}

As Tabelas 2 a 6 ilustram os resultados encontrados nas determinações físico-químicas da garapa nos diferentes tempos de armazenamento e nas diferentes concentrações do antioxidante.

\section{TABELA 2 - VALORES DE pH NOS DIFERENTES TRATAMENTOS DA GARAPA CLARIFICADA-ESTABILIZADA EM CADA TEMPO DE ARMAZENAMENTO *}

\begin{tabular}{cccc}
\hline TEMPO/TRATAMENTO & $\mathbf{5 0} \mathbf{~ p m}$ & $\mathbf{1 2 5} \mathbf{~ p p m}$ & $\mathbf{2 0 0} \mathbf{~ p m}$ \\
\hline $\mathbf{0}$ & $3,97^{\mathrm{aD}}$ & $3,93^{\mathrm{aE}}$ & $3,93^{\mathrm{aE}}$ \\
$\mathbf{1 5}$ & $3,96^{\mathrm{aD}}$ & $3,96^{\mathrm{aD}}$ & $3,94^{\mathrm{bD}}$ \\
$\mathbf{3 0}$ & $4,02^{\mathrm{aC}}$ & $4,00^{\mathrm{bC}}$ & $3,97^{\mathrm{cC}}$ \\
$\mathbf{4 5}$ & $4,01^{\mathrm{aC}}$ & $4,02^{\mathrm{aB}}$ & $3,99^{\mathrm{aB}}$ \\
$\mathbf{6 0}$ & $4,04^{\mathrm{cB}}$ & $4,02^{\mathrm{aB}}$ & $3,99^{\mathrm{bB}}$ \\
$\mathbf{6 6}$ & $4,07^{\mathrm{aA}}$ & $4,06^{\mathrm{aA}}$ & $4,03^{\mathrm{aA}}$ \\
\hline
\end{tabular}

* Médias de 3 repetições.

** Médias seguidas pela mesma letra MINúSCULA, em cada LINHA não diferem entre si a $\mathrm{p} \leq 0,05$.

*** Médias seguidas pela mesma letra MAIÚSCULA, em cada COLUNA não diferem entre si a $p \leq 0,05$.

Os valores de $\mathrm{pH}$ entre as amostras praticamente não diferiram entre si em todos os tempos de armazenamento. Tais valores apresentaram 
aumento para as 3 amostras até o final do período de estocagem, provavelmente, devido à redução nos teores de ácido ascórbico (que contribui para a acidez da bebida).

As amostras praticamente não diferiram entre si em cada tempo de estocagem quanto ao teor de sólidos solúveis (Tabela 3). No entanto, os valores de cada amostra diferiram bastante durante o tempo de armazenamento, talvez pela falta de padronização do teor de sólidos solúveis (Brix) do caldo-de-cana. O mesmo ocorreu para acidez, ou seja, entre si as amostras não diferiram estatisticamente, apenas individualmente houve diferença significativa entre os tempos de armazenamento (Tabela 4).

TABELA 3 - TEORES DE SÓLIDOS SOLÚVEIS (A $20^{\circ} \mathrm{C}$ ) NOS DIFERENTES TRATAMENTOS DA GARAPA CLARIFICADA-ESTABILIZADA EM CADA TEMPO DE ARMAZENAMENTO*

\begin{tabular}{cccc}
\hline TEMPO/TRATAMENTO & $\mathbf{5 0} \mathbf{~ p p m}$ & $\mathbf{1 2 5} \mathbf{~ p p m}$ & $\mathbf{2 0 0} \mathbf{p p m}$ \\
\hline $\mathbf{0}$ & $23,0^{\mathrm{aA}}$ & $23,0^{\mathrm{aC}}$ & $23,0^{\mathrm{aB}}$ \\
$\mathbf{1 5}$ & $23,2^{\mathrm{aBC}}$ & $23,2^{\mathrm{aB}}$ & $22,8^{\mathrm{bC}}$ \\
$\mathbf{3 0}$ & $23,3^{\mathrm{aBC}}$ & $23,5^{\mathrm{aA}}$ & $23,1^{\mathrm{aB}}$ \\
$\mathbf{4 5}$ & $23,2^{\mathrm{aAB}}$ & $23,2^{\mathrm{aB}}$ & $23,0^{\mathrm{bB}}$ \\
$\mathbf{6 0}$ & $23,4^{\mathrm{aC}}$ & $23,5^{\mathrm{aA}}$ & $23,4^{\mathrm{AA}}$ \\
$\mathbf{6 6}$ & $23,4^{\mathrm{aBC}}$ & $23,4^{\mathrm{aA}}$ & $23,4^{\mathrm{aA}}$ \\
\hline
\end{tabular}

* Médias de 3 repetições.

** Médias seguidas pela mesma letra minúscula, em cada linha não diferem entre si a $\mathrm{p} \leq 0,05$.

*** Médias seguidas pela mesma letra maiúscula, em cada coluna não diferem entre si a $\mathrm{p} \leq 0,05$.

TABELA 4 - ACIDEZ TOTAL TITULÁVEL (\% ÁCIDO CÍTRICO) NOS DIFERENTES TRATAMENTOS DA GARAPA CLARIFICADA-ESTABILIZADA EM CADA TEMPO DE ARMAZENAMENTO*

\begin{tabular}{cccc}
\hline TEMPO/TRATAMENTO & $\mathbf{5 0} \mathbf{p p m}$ & $\mathbf{1 2 5} \mathbf{p p m}$ & $\mathbf{2 0 0} \mathbf{p p m}$ \\
\hline $\mathbf{0}$ & $0,230^{\mathrm{aA}}$ & $0,218^{\mathrm{Ba}}$ & $0,230^{\mathrm{aA}}$ \\
$\mathbf{1 5}$ & $0,237^{\mathrm{aA}}$ & $0,218^{\mathrm{bA}}$ & $0,211^{\mathrm{bB}}$ \\
$\mathbf{3 0}$ & $0,186^{\mathrm{aB}}$ & $0,205^{\mathrm{aB}}$ & $0,198^{\mathrm{aBC}}$ \\
$\mathbf{4 5}$ & $0,192^{\mathrm{abB}}$ & $0,179^{\mathrm{bC}}$ & $0,198^{\mathrm{aC}}$ \\
$\mathbf{6 0}$ & $0,192^{\mathrm{aB}}$ & $0,173^{\mathrm{bCD}}$ & $0,198^{\mathrm{aBC}}$ \\
$\mathbf{6 6}$ & $0,173^{\mathrm{bC}}$ & $0,173^{\mathrm{bD}}$ & $0,192^{\mathrm{aC}}$ \\
\hline
\end{tabular}

* Médias de 3 repetições.

** Médias seguidas pela mesma letra minúscula, em cada linha não diferem entre si a $\mathrm{p} \leq 0,05$.

*** Médias seguidas pela mesma letra maiúscula, em cada coluna não diferem entre si a $\mathrm{p} \leq 0,05$. 
Em função do que ocorreu com o teor de sólidos solúveis e com a acidez total titulável não foi verificada diferença significativa entre as amostras para o ratio, mas entre os tempos de armazenamento analisando-se cada amostra (Tabela 5). Notou-se tendência de aumento do ratio do tempo 0 para 66 dias, devido à queda na acidez.

\section{TABELA 5 - VALORES DO RATIO (RELAÇÃO BRIX/ACIDEZ) NOS DIFERENTES TRATAMENTOS DA GARAPA CLARIFICADA-ESTABILIZADA EM CADA TEMPO DE ARMAZENAMENTO*}

\begin{tabular}{cccc}
\hline TEMPO/TRATAMENTO & $\mathbf{5 0} \mathbf{~ p ~ p ~}$ & $\mathbf{1 2 5} \mathbf{p p m}$ & $\mathbf{2 0 0} \mathbf{p p m}$ \\
\hline $\mathbf{0}$ & $100,00^{\mathrm{a}}$ & $105,50^{\mathrm{aE}}$ & $100,00^{\mathrm{aD}}$ \\
$\mathbf{1 5}$ & $97,89^{\mathrm{cE}}$ & $106,42^{\mathrm{bD}}$ & $106,33^{\mathrm{aCD}}$ \\
$\mathbf{3 0}$ & $125,27^{\mathrm{aD}}$ & $114,63^{\mathrm{aCD}}$ & $116,67^{\mathrm{aBC}}$ \\
$\mathbf{4 5}$ & $120,83^{\mathrm{cC}}$ & $129,61^{\mathrm{bC}}$ & $116,16^{\mathrm{aAB}}$ \\
$\mathbf{6 0}$ & $121,87^{\mathrm{aB}}$ & $135,84^{\mathrm{aB}}$ & $118,18^{\mathrm{aA}}$ \\
$\mathbf{6 6}$ & $132,95^{\mathrm{bA}}$ & $132,95^{\mathrm{bA}}$ & $119,79^{\mathrm{aA}}$ \\
\hline
\end{tabular}

* Médias de 3 repetições.

** Médias seguidas pela mesma letra minúscula, em cada linha não diferem entre si a $\mathrm{p} \leq 0,05$.

*** Médias seguidas pela mesma letra maiúscula, em cada coluna não diferem entre si a $p \leq 0,05$.

O teor de ácido ascórbico sofreu queda até o final do período de estocagem sob refrigeração, sendo que os valores encontrados para as três amostras mostraram-se estatisticamente diferentes nos diversos tempos de armazenamento (Tabela 6).

TABELA 6 - TEORES DE VITAMINA C (mg ác. asc./100 mL amostra) NOS DIFERENTES TRATAMENTOS DA GARAPA CLARIFICADA-ESTABILIZADA EM CADA TEMPO DE ARMAZENAMENTO*

\begin{tabular}{|c|c|c|c|}
\hline TEMPO/TRATAMENTO & 50 ppm & 125 ppm & 200 ppm \\
\hline 0 & $4,50^{\mathrm{CA}}$ & $10,91^{\mathrm{bA}}$ & $19,54^{\text {aA }}$ \\
\hline 15 & $3,35^{c B}$ & $10,52^{\mathrm{bB}}$ & $19,04^{\text {aB }}$ \\
\hline 30 & $2,39^{\mathrm{cc}}$ & $9,32^{\mathrm{bc}}$ & $18,64^{\mathrm{aBC}}$ \\
\hline 45 & $2,10^{\mathrm{cD}}$ & $8,95^{b D}$ & $18,41^{\mathrm{ac}}$ \\
\hline 60 & $1,96^{\mathrm{cE}}$ & $8,82^{b E}$ & $18,17^{\mathrm{aD}}$ \\
\hline 66 & $1,77^{\mathrm{cF}}$ & $8,76^{b F}$ & $17,88^{a E}$ \\
\hline
\end{tabular}

* Médias de 3 repetições.

** Médias seguidas pela mesma letra minúscula, em cada linha não diferem entre si a $\mathrm{p} \leq 0,05$.

${ }^{* * \star}$ Médias seguidas pela mesma letra maiúscula, em cada coluna não diferem entre si a $\mathrm{p} \leq 0,05$. 
Para o tratamento 1, a perda total de vitamina $\mathrm{C}$ em relação ao teor adicionado foi de $64,6 \%$. Após o processamento, a diminuição no teor de ácido ascórbico foi de $10 \%$, ou seja, passou de $5 \mathrm{mg} / 100 \mathrm{~mL}$ (adicionado) para $4,50 \mathrm{mg} / 100 \mathrm{~mL}$. Do tempo 0 para 66 dias, a queda foi de $60,7 \%$ (de $4,50 \mathrm{mg} / 100 \mathrm{~mL}$ para $1,77 \mathrm{mg} / 100 \mathrm{~mL}$ ). No tratamento 2 , a perda total de vitamina C foi de $30 \%$. A diminuição no teor de ácido ascórbico foi de $12,7 \%$, após o processamento, ou seja, passou de $12,5 \mathrm{mg} / 100 \mathrm{~mL}$ (adicionado) para $10,91 \mathrm{mg} / 100 \mathrm{~mL}$. A perda desse elemento foi de $19,7 \%$ do tempo 0 para 66 dias (de 10,91 mg/100 mL para $8,76 \mathrm{mg} / 100 \mathrm{~mL}$ ). A perda total de vitamina C para o tratamento 3 foi de 10,6\%. Após o processamento, a diminuição no teor de vitamina $\mathrm{C}$ foi de 2,3\%, ou seja, passou de $20 \mathrm{mg} / 100 \mathrm{~mL}$ (adicionado) para $19,54 \mathrm{mg} / 100 \mathrm{~mL}$. Entre os tempos 0 e 66 dias, o decréscimo foi de $8,5 \%$ (de 19,54 mg/100 mL para 17,88 mg/100 mL).

A vitamina $C$ oxida rapidamente em solução aquosa por processo enzimático e não-enzimático, especialmente quando exposta ao ar, ao calor e à luz, sendo a reação acelerada por íons metálicos $\left(\mathrm{Cu}^{++} \mathrm{e}\right.$ $\mathrm{Fe}^{++}$) (ARAÚJO, 1995).

A principal forma de degradação de vitamina $C$ em produtos processados envolve basicamente reações aeróbica e anaeróbica de natureza não-enzimática. Estudos demonstraram que em produtos de frutas com $\mathrm{pH}<4,0$, o escurecimento não-enzimático ocorre principalmente devido à degradação do ácido ascórbico. A velocidade da degradação depende de fatores como concentração da vitamina, $\mathrm{pH}$, conteúdo de oxigênio, temperatura de estocagem e processamento (OLIVA, 1995).

As condições aeróbicas são proporcionadas pela incorporação de ar durante as etapas de extração, filtração, mistura e enchimento de embalagens. Na presença do oxigênio, o ácido ascórbico é oxidado a ácido dehidroascórbico, sendo esse hidrolisado e oxidado a ácido dicetogulônico e ácido oxálico. A velocidade da oxidação aeróbica depende do $\mathrm{pH}$, sendo o meio alcalino o mais favorável (ARAÚJO, 1995; HENSHALL, 1981; OLIVA, 1995).

A presença de oxigênio foi o fator que mais colaborou para a queda no teor do ácido ascórbico durante o processamento, já que a desaeração do produto não foi realizada por falta de equipamento apropriado. 
É pouco provável que tenha ocorrido degradação enzimática do ácido ascórbico, durante o armazenamento, pela ação das enzimas citocromo oxidase, ácido ascórbico oxidase e peroxidase, devido ao tratamento térmico do produto (inativação das enzimas pelo calor). Portanto, a queda nos teores de vitamina $\mathrm{C}$ poderia ser explicada pela ação da luz (embalagem transparente) e pela ação do oxigênio incorporado durante o processamento.

As Figuras 11, 12 e 13 ilustram a análise de regressão da determinação do teor de ácido ascórbico para cada amostra durante o período de estocagem.

\section{FIGURA 11 - ANÁLISE DE REGRESSÃO CONTENDO A EQUAÇÃO DA RETA E O VALOR DE R² PARA A AMOSTRA 1 (50 ppm)}

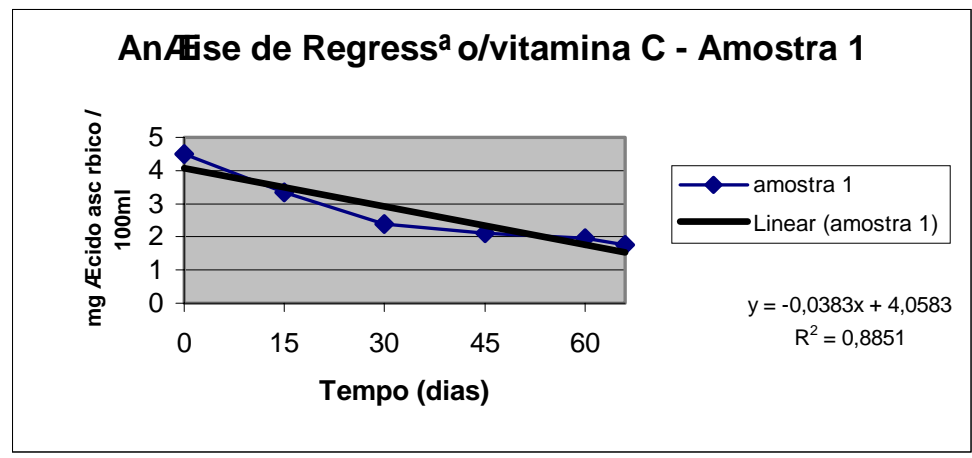

As 3 amostras apresentaram o mesmo comportamento, confirmando os resutados da análise sensorial.

ROING et al. (1999) estudaram a retenção de ácido ascórbico em suco de laranja processado e envasado em embalagens cartonadas, tendo elaborado a curva de regressão entre o teor de ácido ascórbico retido e o tempo de estocagem. Observaram que após 2-3 meses de armazenamento houve pouca correlação (baixo valor de $R^{2}$ ) entre o teor desse constituinte e o tempo de armazenamento (em temperatura ambiente ou a $5^{\circ} \mathrm{C}$ ). Após 3 meses de estocagem, o teor de vitamina $\mathrm{C}$ manteve-se constante até o final de 12 meses. 
FIGURA 12 - ANÁLISE DE REGRESSÃO CONTENDO A EQUAÇÃO DA RETA E O VALOR DE R² PARA A AMOSTRA 2 (125 ppm)

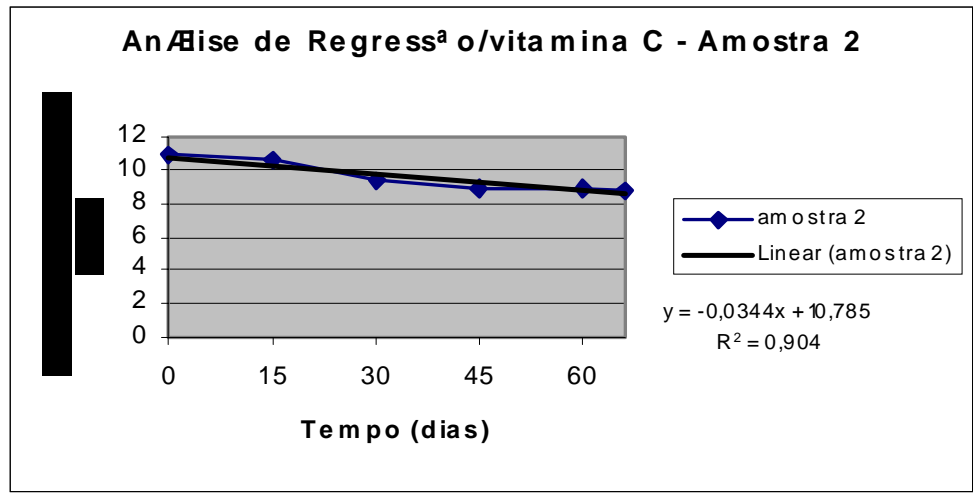

FIGURA 13 - ANÁLISE DE REGRESSÃO CONTENDO A EQUAÇÃO DA RETA E O VALOR DE R² PARA A AMOSTRA 3 (200 ppm)

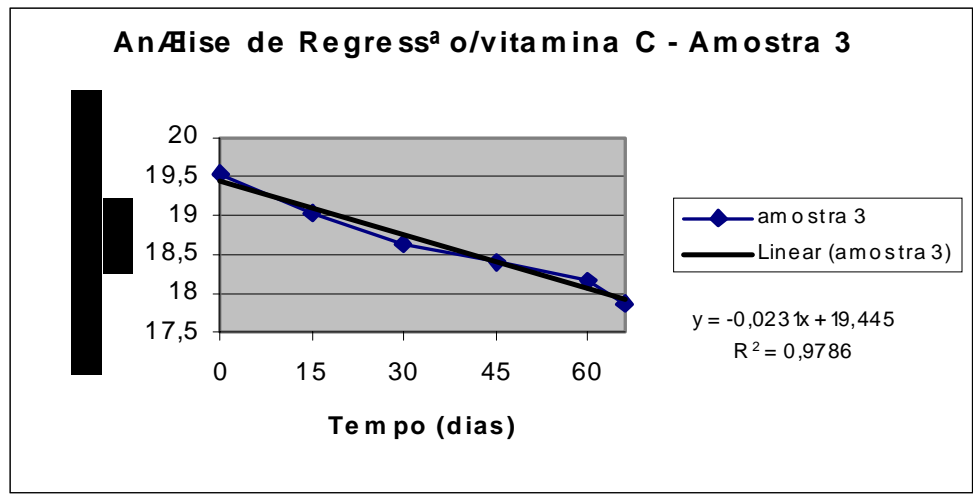

\section{CONCLUSÃO}

Conclui-se em relação à Análise de Regressão que não houve correlação entre os tempos de armazenamento e os atributos para todas as 
amostras, ou seja, as amostras mantiveram sua qualidade sensorial até os 66 dias de estocagem sob refrigeração.

Quanto ao teor de vitamina C, as amostras diferiram entre si em todos os tempos estudados, devido justamente aos diferentes tratamentos. Para todas as amostras ocorreu redução nos níveis desse constituinte durante o armazenamento, em função de sua degradação.

Apesar de não ter ocorrido diferença significativa entre as amostras em relação aos atributos estudados, nos diferentes tempos de estocagem, o tratamento 2 foi considerado o melhor. Seu nível intermediário de ácido ascórbico torna o produto menos dispendioso.

\section{Abstract \\ STUDY OF THE INTERFERENCE OF THREE DIFFERENTS ASCORBIC ACID CONCENTRATIONS IN THE PHYSICAL, CHEMICAL AND SENSORY QUALITY OF PARTIALLY CLARIFIED-STABILIZED SUGAR CANE JUICE STORED UNDER REFRIGERATION}

Three distinct concentrations of ascorbic acid (50 ppm - sample 1; 125 ppm sample 2; 200 ppm - sample 3) on the sensory quality of partially clarified-stabilized sugar cane juice, subsequently acidified and added of preservative before pasteurization $\left(82^{\circ} \mathrm{C} / 15 \mathrm{seg}\right.$.) were tested. After heat treatment the beverage was cooled, bottled in PET and stored under refrigeration (4-6ㅇ) for two months. In addition to the sensory analyses, physical-chemical analyses $\left(\mathrm{pH}\right.$, ${ }^{\circ}$ Brix, acidity, ratio, ascorbic acid content) were carried out at each storage time. The vitamin $\mathrm{C}$ determinations showed that this constituent decreased with storage time. The sensory acceptance test showed that the three samples maintained their sensory quality during the storage period. Thus sample 2 was chosen as the best product since an intermediate concentration of ascorbic acid was added to this sample, being therefore less costly.

KEY-WORDS: SUGAR CANE SENSORY EVALUATION; SUGAR CANE JUICE.

\section{REFERÊNCIAS}

1 ABREU, L.E.V.; SCHMITZ, C.M. Teor de ácido ascórbico em alimentos de origem vegetal. Revista do Centro de Ciências Rurais, v.1, n.4, p.69-71, 1971.

2 ALVES, R.M.V.; GARCIA, E.E.C. Embalagem para sucos de frutas. Coletânea do Instituto de Tecnologia de Alimentos, v.23, n.2, p.105-122, jul./dez. 1993.

3 ARAÚJO, J.M.A. Química de Alimentos: teoria e prática. Viçosa: Universidade Federal de Viçosa, 1995. 335 p. 

analysis of AOAC Internacional. $12^{\text {th }}$ ed. Washington, 1984. p.844-845.

5 AOAC. Association of Official Analytical Chemists. Official methods of analysis of AOAC Internacional. $16^{\text {th }}$ ed. Washington, 1997. V. II, cap.37: Fruits and fruit products.

6 ASTM. American Society for Testing and Materials. Standard guide for the shelf life determination of consumer products by sensory evaluation. Philadelphia, 1993. 10 p. (ASTM E 18.06.07)

7 BENASSI, M.T. Análise dos efeitos de diferentes parâmetros na estabilidade de vitamina C em vegetais processados. Campinas 1990. 159 p. Dissertação (Mestrado), Faculdade de Engenharia de Alimentos, UNICAMP.

8 CORREAA NETO, R.S. Processamento de suco de laranja pasteurizado em garrafas de polietileno tereftalato (PET). Campinas, 1998. 93 p. Dissertação (Mestrado), Faculdade de Engenharia de Alimentos, UNICAMP.

9 EVANGELISTA, J. Tecnologia de alimentos. 2.ed. São Paulo: Atheneu, 1994. $652 \mathrm{p}$.

10 FENNEMA, O.R. Principles of food science: part 2: physical principles of food preservation. New York: Marcel Dekker, 1975.

11 GRAUMLICH, T.R.; MARCY, J.E.; ADAMS, J.P. Asseptically packaged orange juice and concentrate: a review of the influence of processing and packaging conditions on quality. Journal of Agricultural and Food Chemistry, v.34, n.3, p. 402-405, 1986.

12 HENSHALL, J.D. Ascorbic acid in fruit juices and beverages. In: COUNSELL, J.N.; HORNIG, D.H. Vitamin C: ascorbic acid. London: Applied Science Publishers, 1981. Cap.8, p.123-137.

13 INSTITUTO ADOLFO LUTZ. Normas Analíticas do Instituto “Adolfo Lutz". Métodos químicos e físicos para análise de alimentos. 2.ed. São Paulo, 1976. $371 \mathrm{p}$.

14 LEITÃO, M.F.F. Microbiologia de sucos e produtos ácidos. Boletim do Instituto de Tecnologia de Alimentos, n.33, p.9-42, mar. 1973.

15 MARTIN, J.J.; SOLANES, E.; BOTA, E.; SANCHO, J. Evolucion quimica y organoleptica del zumo de naranja pasterizado. Alimentaria, p.59-63, abr. 1995.

16 MEILGAARD, M.; CIVILLE, G.V.; CARR, B.T. Sensory evaluation techniques. New YorK: CRC Press, 1987. 281 p.

17 OLIVA, P.B. Estudo do armazenamento da acerola in natura e estabilidade do néctar de acerola. Campinas, 1995. 103 p. Dissertação (Mestrado) - Faculdade de Engenharia de Alimentos, UNICAMP. 
PRATI, P.; MORETTI, R.H. Desenvolvimento de processo para clarificação de caldo-de-cana para consumo. In: CONGRESSO BRASILEIRO DE CIÊNCIA E TECNOLOGIA DE ALIMENTOS, 18., Porto Alegre, 2002. Anais... Porto Alegre, 2002.

19 ROING, M.G.; BELLO, J.F.; RIVERA, Z.S.; KENNEDY, J.F. Studies on the occurrence of non-enzymatic browning during storage of citrus juice. Food Research Internacional, n.32, p.609-619, 1999.

20 SAS Institute. SAS User's Guide: statistics. Cary, USA, 1993.

21 SHAW, P.E.; MOSHONAS, M.G. Ascorbic acid retention in orange juice stored under simulated consumer home conditions. Journal of Food Science, v.56, n.3, p.867-868, 1991.

22 SIMÃO, A.M. Aditivos para alimentos sob o aspecto toxicológico. 2.ed. São Paulo: Nobel, 1985. 274 p.

23 SOCCOL, C.R.; SCHWAB, A.; KATAOKA, C.E. Avaliação microbiológica do caldo-de-cana (garapa) na cidade de Curitiba. Boletim do Centro de Pesquisa e Processamento de Alimentos, v.8, n.2, p.116-125, jul./dez. 1990.

24 STATISTICA for windows: version 5.0. Tulsa, 1995.

25 STONE, H.; SIDEL, J.L. Sensory evaluation practices. New York: Academic Press, 1993. $338 \mathrm{p}$.

26 TOCCHINI, R.P. Efeito da temperatura e do tempo de armazenamento na qualidade do suco concentrado de laranja pasteurizado embalado assepticamente em Tetra-Brik. Piracicaba, 1985. 51 p. Dissertação (Mestrado), Escola Superior de Agricultura "Luiz de Queiroz, USP.

27 YUSOF, S.; SHIAN, L.S.; OSMAN, A. Changes in quality of sugar-cane juice upon delayed extraction and storage. Food Chemistry, v.68, p.395-401, 2000. 